\title{
ON THE RANGE OF THE Y-TRANSFORM
}

\author{
Vu KIM TUaN
}

The ranges of the Y-integral transform in some spaces of functions are described.

\section{INTRODUCTION}

The $\mathrm{Y}$-transform $Y_{\nu}$ is defined by $[8,6]$

$$
f(x)=\left(Y_{\nu} g\right)(x)=\int_{0}^{\infty} \sqrt{x y} Y_{\nu}(x y) g(y) d y, \quad x \in R_{+}=(0, \infty)
$$

if the integral converges in some sense (absolutely, improper, mean convergence), where $Y_{\nu}(x)$ is the Bessel function of the second kind [1]. The Y-transform $Y_{\nu}$ has been considered in $\mathcal{L}_{\mu, p}$ in $[3,6,7]$. In particular, it follows that in $L_{2}\left(R_{+}\right)=\mathcal{L}_{1 / 2,2}$ the Y-transform $Y_{\nu}$ is bounded if $|\operatorname{Re} \nu|<1$, and if, moreover, $0<|\mathcal{R} e \nu|<1$, then the range of the $Y$-transform $Y_{\nu}$ is $L_{2}\left(R_{+}\right)$:

$$
\begin{aligned}
\left\|Y_{\nu} g\right\|_{L_{2}\left(R_{+}\right)} & \leqslant C\|g\|_{L_{2}\left(R_{+}\right)}, & & |\mathcal{R} e \nu|<1, \\
\|g\|_{L_{2}\left(R_{+}\right)} & \leqslant C\left\|Y_{\nu} g\right\|_{L_{2}\left(R_{+}\right)}, & & 0<|\mathcal{R} e \nu|<1,
\end{aligned}
$$

where $C$ is an independent constant, (but different in distinct inequalities). The $\mathbf{H}$ transform $\mathbf{H}_{\nu}[8,6]$ denoted by

$$
g(x)=\left(\mathbf{H}_{\nu} f\right)(x)=\int_{0}^{\infty} \sqrt{x y} \mathbf{H}_{\nu}(x y) f(y) d y, \quad x \in R_{+},
$$

is the inverse of $\mathrm{Y}$-transform $Y_{\nu}$ in $L_{2}\left(R_{+}\right)$if $-1<\mathcal{R e} \nu<0$. If $0<\mathcal{R} e \nu<1$ the inverse formula (4) should be replaced by formula (51) or, equivalently, (52). Here $\mathbf{H}_{\nu}(x)$ is the Struve function [1]. The $\mathrm{Y}$ - and $\mathbf{H}$-transforms are of importance in many singular axially symmetric potential problems [6]. In this work we describe precisely the range of the Y-transform in some spaces of functions. The range of the Y-transform of functions with compact supports (analogous to the Paley-Wiener theorem for the Fourier transform [5]) is also considered. It is worth remarking that our Paley-Wiener

Received 22nd November, 1996

Supported by the Kuwait University research grant SM 112.

Copyright Clearance Centre, Inc. Serial-fee code: 0004-9729/96 \$A2.00+0.00. 
theorem (Theorem 2) is different from the classical ones describing Fourier transform of compactly supported functions in terms of entire functions of exponential type [5]. (For the Hankel transform of compactly supported functions see [4].) The theorem stated here involves the spectral radius [12] of some differential operator obtained from the Bessel differential equation and having the kernel of the Y-transform as "eigenfunctions", (similar ideas have been applied in $[2,11]$ to the Fourier transform). Nevertheless, its proof is straightforward, without referring to spectral theory. Since the H-transform $\mathbf{H}_{\nu}$ is the inverse of the $\mathrm{Y}$-transform $Y_{\nu}$ in all spaces we considered in this paper, corresponding theorems on the range of the $\mathbf{H}$-transform can be easily derived.

\section{Y-Transform of Polynomial Decreasing Functions}

We describe the range of the $Y$-transform on the space of functions $g(y)$ square integrable together with $y^{n} g(y), n=1,2, \ldots$ (polynomial decreasing functions):

THEOREM 1. A function $f(x)$ is the $Y$-transform $Y_{\nu}, 0<|\mathcal{R} e \nu|<1 / 2$, of a function $g(y)$, square integrable together with $y^{n} g(y), n=1,2, \ldots$, if and only if

(i) $f(x)$ is infinitely differentiable on $R_{+}$;

(ii) $\left(d^{2} / d x^{2}+\left(1 / x^{2}\right)\left((1 / 4)-\nu^{2}\right)\right)^{n} f(x), \quad n=0,1, \ldots$, belongs to $L_{2}\left(R_{+}\right)$;

(iii) $\left(d^{2} / d x^{2}+\left(1 / x^{2}\right)\left((1 / 4)-\nu^{2}\right)\right)^{n} f(x), \quad n=0,1, \ldots$, tends to 0 as $x$ tends both to 0 and to infinity;

(iv) $x(d / d x)\left(d^{2} / d x^{2}+\left(1 / x^{2}\right)\left((1 / 4)-\nu^{2}\right)\right)^{n} f(x), \quad n=0,1, \ldots, \quad$ is bounded at 0 ;

(v) $(d / d x)\left(d^{2} / d x^{2}+\left(1 / x^{2}\right)\left((1 / 4)-\nu^{2}\right)\right)^{n} f(x), \quad n=0,1, \ldots$, tends to 0 as $x$ tends to infinity;

(vi) The improper integrals

$$
\int_{\rightarrow 0}^{\rightarrow \infty} x^{\nu-1 / 2} \frac{d^{2}}{d x^{2}}+\frac{1}{x^{2}}\left(\frac{1}{4}-\nu^{2}\right)^{n} f(x) d x
$$

exist and vanish for all $n=1,2, \ldots$, as well as for $n=0$ if $-1 / 2<$ $\mathcal{R e} \nu<0$.

Proof: (a) Let $y^{n} g(y)$ belong to $L_{2}\left(R_{+}\right)$for all $n=0,1,2, \ldots$, then $y^{n} g(y)$ belongs to $L_{1}\left(R_{+}\right)$for all $n=0,1,2, \ldots$ Let $f(x)$ be the Y-transform $Y_{\nu}, 0<$ $|\operatorname{Re} \nu|<1 / 2$, of $g(y)$ (the Y-transform $Y_{\nu}$ of $g(y)$ with other values of $\nu$ also appears in the proof, but it is not denoted by $f(x))$.

(a-i) We have [1]

$$
\frac{d^{n}}{d x^{n}} Y_{\nu}(x)=2^{-n} \sum_{j=0}^{n}(-1)^{j}\left(\begin{array}{c}
n \\
j
\end{array}\right) Y_{\nu-n+2 j}(x)
$$


Therefore,

$$
\begin{aligned}
\frac{\partial^{n}}{\partial x^{n}}\left(\sqrt{x y} Y_{\nu}(x y)\right)=\sum_{k=0}^{n} & \sum_{j=0}^{k}(-1)^{n+j-k} 2^{-k}(-1 / 2)_{n-k}\left(\begin{array}{l}
n \\
k
\end{array}\right)\left(\begin{array}{l}
k \\
j
\end{array}\right) \\
& x^{1 / 2+k-n} y^{1 / 2+k} Y_{\nu-k+2 j}(x y),
\end{aligned}
$$

where $(a)_{n}=\Gamma(a+n) / \Gamma(a)$ is the Pochhammer symbol [1]. The Bessel function of the second kind $Y_{\nu}(y)$ has the asymptotics $[1]$

$$
Y_{\nu}(y)= \begin{cases}\sqrt{\frac{2}{\pi y}}\left[\sin \left(y-\frac{\nu \pi}{2}-\frac{\pi}{4}\right)+\frac{4 \nu^{2}-1}{8 y} \cos \left(y-\frac{\nu \pi}{2}-\frac{\pi}{4}\right)\right]+O\left(y^{-5 / 2}\right), & y \rightarrow \infty \\ O\left(y^{-|\operatorname{Re} \nu|}\right), & y \rightarrow 0 .\end{cases}
$$

Consequently, $\frac{\partial^{n}}{\partial x^{n}}\left[\sqrt{x y} Y_{\nu}(x y)\right], \quad|\mathcal{R} e \nu|<1$, as a function of $y$ has the asymptotics $O\left(y^{1 / 2-|R e \nu|}\right)$ in the neighbourhood of 0 and $O\left(y^{n}\right)$ at infinity. Hence, $\frac{\partial^{n}}{\partial x^{n}}\left[\sqrt{x y} Y_{\nu}(x y)\right] g(y),|\mathcal{R} e \nu|<1$, as a function of $y$ belongs to $L_{1}\left(R_{+}\right)$for all $n=0,1,2, \ldots$, and therefore, $f(x)$ is infinitely differentiable on $R_{+}$.

(a-ii) Since $Y_{\nu}(x)$ satisfies the Bessel differential equation [1]

$$
x^{2} u^{\prime \prime}+x u^{\prime}+\left(x^{2}-\nu^{2}\right) u=0
$$

the function $\sqrt{x} Y_{\nu}(x)$ is a solution of the equation

$$
x^{2} u^{\prime \prime}+\left(x^{2}+\frac{1}{4}-\nu^{2}\right) u=0
$$

Therefore, we have

$$
\left[\frac{\partial^{2}}{\partial x^{2}}+\frac{1}{x^{2}}\left(\frac{1}{4}-\nu^{2}\right)\right]^{n}\left(\sqrt{x y} Y_{\nu}(x y)\right)=\left(-y^{2}\right)^{n} \sqrt{x y} Y_{\nu}(x y)
$$

Consequently,

(11)

$$
\left[\frac{d^{2}}{d x^{2}}+\frac{1}{x^{2}}\left(\frac{1}{4}-\nu^{2}\right)\right]^{n} f(x)=(-1)^{n} \int_{0}^{\infty} \sqrt{x y} Y_{\nu}(x y) y^{2 n} g(y) d y, \quad \mid \mathcal{R} \text { e } \nu \mid<1 / 2
$$

By using inequality (2) for the Y-transform (11) of $y^{2 n} g(y) \in L_{2}\left(R_{+}\right)$, we obtain that $\left[d^{2} / d x^{2}+\left(1 / x^{2}\right)\left((1 / 4)-\nu^{2}\right)\right]^{n} f(x),|\mathcal{R} e \nu|<1 / 2, n=0,1, \ldots$, belongs to $L_{2}\left(R_{+}\right)$. 
(a-iii) From (7) we see that the function $\sqrt{x y} Y_{\nu}(x y),|\operatorname{Re} \nu|<1 / 2$, has the asymptotics $x^{1 / 2-\left|R_{e} \nu\right|}$ as $x$ tends to 0 , and is uniformly bounded on $R_{+}$. Because $y^{2 n} g(y) \in L_{1}\left(R_{+}\right)$, by applying the dominated convergence theorem [12] we have

$$
\lim _{x \rightarrow 0}\left[\frac{d^{2}}{d x^{2}}+\frac{1}{x^{2}}\left(\frac{1}{4}-\nu^{2}\right)\right]^{n} f(x)=(-1)^{n} \int_{0}^{\infty} \lim _{x \rightarrow 0}\left[\sqrt{x y} Y_{y}(x y)\right] y^{2 n} g(y) d y=0,
$$

$$
|\mathcal{R} e \nu|<1 / 2 \text {. }
$$

Since $\sqrt{x y} Y_{\nu}(x y),|\operatorname{Re} \nu|<3 / 2$, is uniformly bounded for $x, y \in[1, \infty)$ and $y^{n} g(y) \in$ $L_{1}\left(R_{+}\right)$, for every $\varepsilon>0$ and for every $n, n=0,1, \ldots$, one can choose $b$ large enough so that

$$
\left|\int_{b}^{\infty} \sqrt{x y} Y_{\nu}(x y) y^{n} g(y) d y\right|<\varepsilon, \quad|\operatorname{Re} \nu|<3 / 2,
$$

uniformly with respect to $x \in[1, \infty)$. On the other hand, from (7) one can conclude that the integral

$$
\int_{a x}^{b x} \sqrt{y} Y_{\nu}(y) d y, \quad|\mathcal{R} e \nu|<1 / 2,
$$

is uniformly bounded for all non-negative $a, b$ and $x$. Hence,

$$
\int_{a}^{b} \sqrt{x y} Y_{\nu}(x y) d y=\frac{1}{x} \int_{a x}^{b x} \sqrt{y} Y_{\nu}(y) d y, \quad|\mathcal{R} e \nu|<1 / 2,
$$

tends to 0 uniformly in $a, b$ for $0 \leqslant a<b<\infty$ as $x$ tends to infinity. Consequently, applying the generalised Riemann-Lebesgue lemma [8] we get

$$
\lim _{x \rightarrow \infty} \int_{0}^{b} \sqrt{x y} Y_{\nu}(x y) y^{2 n} g(y) d y=0, \quad 0<b<\infty,|\mathcal{R} e \nu|<1 / 2 .
$$

Because $\varepsilon$ can be taken arbitrarily small, from (13) and (16) we obtain

$$
\lim _{x \rightarrow \infty} \int_{0}^{\infty} \sqrt{x y} Y_{\nu}(x y) y^{2 n} g(y) d y=0, \quad|\mathcal{R} e \nu|<1 / 2 .
$$

Hence,

$$
\lim _{x \rightarrow \infty}\left[\frac{d^{2}}{d x^{2}}+\frac{1}{x^{2}}\left(\frac{1}{4}-\nu^{2}\right)\right]^{n} f(x)=0, \quad n=0,1, \ldots,|\operatorname{Re} \nu|<1 / 2 .
$$

(a-iv) Since [1]

$$
2 \frac{d}{d x}\left(\sqrt{x} Y_{\nu}(x)\right)=\sqrt{x} Y_{\nu-1}(x)-\sqrt{x} Y_{\nu+1}(x)+\frac{1}{\sqrt{x}} Y_{\nu}(x),
$$


we have

$$
\begin{aligned}
& \frac{d}{d x}\left[\frac{d^{2}}{d x^{2}}+\frac{1}{x^{2}}\left(\frac{1}{4}-\nu^{2}\right)\right]^{n} f(x)=\frac{(-1)^{n}}{2} \int_{0}^{\infty} \sqrt{x y} Y_{\nu-1}(x y) y^{2 n+1} g(y) d y \\
& +\frac{(-1)^{n+1}}{2} \int_{0}^{\infty} \sqrt{x y} Y_{\nu+1}(x y) y^{2 n+1} g(y) d y+\frac{(-1)^{n}}{2 x} \int_{0}^{\infty} \sqrt{x y} Y_{\nu}(x y) y^{2 n} g(y) d y
\end{aligned}
$$

The function $\sqrt{x} Y_{\mu}(x)$ is uniformly bounded on $[1, \infty)$, and is of the order $O\left(x^{1 / 2-|R e \mu|}\right)$ on $(0,1)$. Therefore, for $x \in(0,1)$,

$$
\begin{aligned}
\left|\int_{0}^{\infty} \sqrt{x y} Y_{\mu}(x y) g(y) d y\right| & \leqslant\left|\int_{0}^{1 / x} \sqrt{x y} Y_{\mu}(x y) g(y) d y\right|+\left|\int_{1 / x}^{\infty} \sqrt{x y} Y_{\mu}(x y) g(y) d y\right| \\
& \leqslant C x^{1 / 2-|\mathcal{R} e \mu|} \int_{0}^{1 / x} y^{1 / 2-|\mathcal{R} e \mu|}|g(y)| d y+C \int_{1 / x}^{\infty}|g(y)| d y \\
& \leqslant C x^{1 / 2-|\mathcal{R} e \mu|} \int_{0}^{\infty} y^{1 / 2-|\mathcal{R} e \mu|}|g(y)| d y+C \int_{0}^{\infty}|g(y)| d y .
\end{aligned}
$$

Hence, in the neighbourhood of 0 we have

$$
\begin{aligned}
\frac{1}{x} \int_{0}^{\infty} \sqrt{x y} Y_{\nu}(x y) y^{2 n} g(y) d y & =O\left(x^{-1}\right), \\
\int_{0}^{\infty} \sqrt{x y} Y_{\nu-1}(x y) y^{2 n+1} g(y) d y & =O\left(x^{\mathcal{R e} \nu-1 / 2}\right), \\
\int_{0}^{\infty} \sqrt{x y} Y_{\nu+1}(x y) y^{2 n+1} g(y) d y & =O\left(x^{-\mathcal{R} e \nu-1 / 2}\right), \quad|\operatorname{Re} \nu|<1 / 2 .
\end{aligned}
$$

By combining (20) and (22), we obtain

$$
\begin{aligned}
x \frac{d}{d x}\left[\frac{d^{2}}{d x^{2}}+\frac{1}{x^{2}}\left(\frac{1}{4}-\nu^{2}\right)\right]^{n} f(x) & =O(1), \quad x \rightarrow 0 ; \\
n & =0,1, \ldots ;|\operatorname{Re} \nu|<1 / 2 .
\end{aligned}
$$

(a-v) Let $|\mathcal{R e} \nu|<3 / 2$. For every $\varepsilon>0$ choose $b$ so that the inequality (13) holds. Because $(x y)^{3 / 2} Y_{\nu}(x y),|\mathcal{R} e \nu|<3 / 2$, is uniformly bounded for $x, y \in R_{+}, x y \leqslant 1$, then

$$
\left|\int_{0}^{1 / x} \sqrt{x y} Y_{\nu}(x y) y^{n+1} g(y) d y\right| \leqslant \frac{C}{x} \int_{0}^{1 / x} y^{n}|g(y)| d y
$$

Hence,

$$
\lim _{x \rightarrow \infty} \int_{0}^{1 / x} \sqrt{x y} Y_{\nu}(x y) y^{n+1} g(y) d y=0, \quad|\mathcal{R e} \nu|<3 / 2
$$


Let

$$
\Phi(x, y)= \begin{cases}\sqrt{x y} Y_{\nu}(x y) d y, & y>1 / x \\ 0, & y \leqslant 1 / x\end{cases}
$$

Then $\Phi(x, y)$ is uniformly bounded. The integral

$$
\int_{a x}^{b x} \sqrt{y} Y_{\nu}(y) d y, \quad|\mathcal{R} e \nu|<3 / 2
$$

is uniformly bounded for all non-negative $a, b$ and $x$ such that $a x \geqslant 1$. Hence,

$$
\int_{a}^{b} \Phi(x, y) d y=\frac{1}{x} \int_{\max \{1, a x\}}^{b x} \sqrt{y} Y_{\nu}(y) d y, \quad|\mathcal{R} e \nu|<3 / 2
$$

tends to 0 uniformly in $a, b$ for $0 \leqslant a<b<\infty$ as $x$ tends to infinity. Consequently, applying again the generalised Riemann-Lebesgue lemma [8] we get

$$
\lim _{x \rightarrow \infty} \int_{0}^{b} \Phi(x, y) y^{n} g(y) d y=0, \quad 0<b<\infty
$$

This means that

$$
\lim _{x \rightarrow \infty} \int_{1 / x}^{b} \sqrt{x y} Y_{\nu}(x y) y^{n} g(y) d y=0, \quad 0<b<\infty,|\mathcal{R} e \nu|<3 / 2 .
$$

Because $\varepsilon$ can be taken arbitrarily small, from (13), (25) and (30) we obtain

$$
\lim _{x \rightarrow \infty} \int_{0}^{\infty} \sqrt{x y} Y_{\nu}(x y) y^{n+1} g(y) d y=0, \quad n=0,1, \ldots,|\operatorname{Re} \nu|<3 / 2
$$

If $|\mathcal{R} e \nu|<1 / 2$, then $|\mathcal{R} e \nu \mp 1|<3 / 2$. Hence,

$$
\begin{aligned}
& \lim _{x \rightarrow \infty} \int_{0}^{\infty} \sqrt{x y} Y_{\nu-1}(x y) y^{2 n+1} g(y) d y=0, \\
& \lim _{x \rightarrow \infty} \int_{0}^{\infty} \sqrt{x y} Y_{\nu+1}(x y) y^{2 n+1} g(y) d y=0, \quad|\mathcal{R} e \nu|<1 / 2 .
\end{aligned}
$$

Applying now formulas (20), (31) and (32), we have

$$
\lim _{x \rightarrow \infty} \frac{d}{d x}\left[\frac{d^{2}}{d x^{2}}+\frac{1}{x^{2}}\left(\frac{1}{4}-\nu^{2}\right)\right]^{n} f(x)=0, \quad n=0,1, \ldots, \mid \mathcal{R} \text { e } \nu \mid<1 / 2 .
$$

(a-vi) The special case $-1 / 2<\mathcal{R e} \nu<0$ has been proved in [3]. We give here a proof valid for all the range of $\nu$. Integral (11) converges uniformly with respect to $x$ 
on every compact subset of $R_{+}$. Therefore, one can interchange the order of integration in the following formula to obtain

$$
\begin{aligned}
\int_{1 / N}^{N} & x^{\nu-1 / 2}\left[\frac{d^{2}}{d x^{2}}+\frac{1}{x^{2}}\left(\frac{1}{4}-\nu^{2}\right)\right]^{n} f(x) d x \\
& =(-1)^{n} \int_{1 / N}^{N} x^{\nu-1 / 2} \int_{0}^{\infty} \sqrt{x y} Y_{\nu}(x y) y^{2 n} g(y) d y d x \\
& =(-1)^{n} \int_{0}^{\infty} y^{2 n-\nu-1 / 2} g(y) \int_{y / N}^{y N} x^{\nu} Y_{\nu}(x) d x d y, \quad 0<N<\infty .
\end{aligned}
$$

The last inner integral in (34) is uniformly bounded for all nonnegative $N$ and $y$, provided that $|\mathcal{R} e \nu|<1 / 2$. For $y^{2 n-\nu-1 / 2} g(y) \in L_{1}\left(R_{+}\right)$under the restriction $\mathcal{R} e \nu<$ 0 , and $n \geqslant 1$ otherwise, one can apply the dominated convergence theorem to obtain

$$
\begin{aligned}
\lim _{N \rightarrow \infty} \int_{1 / N}^{N} x^{\nu-1 / 2}\left[\frac{d^{2}}{d x^{2}}+\frac{1}{x^{2}}\left(\frac{1}{4}-\nu^{2}\right)\right]^{n} f(x) d x & \\
=(-1)^{n} \int_{0}^{\infty} y^{2 n-\nu-1 / 2} g(y) \int_{0}^{\infty} x^{\nu} Y_{\nu}(x) d x d y, & n=0,1, \ldots ;-1 / 2<\operatorname{Re} \nu<0, \\
& n=1,2, \ldots ; 0 \leqslant \operatorname{Re} \nu<1 / 2 .
\end{aligned}
$$

Applying now the formula [1]

$$
\begin{gathered}
\int_{0}^{\infty} x^{\mu} Y_{\nu}(x) d x=\frac{2^{\mu}}{\pi} \sin \frac{\pi}{2}(\mu-\nu) \Gamma\left(\frac{\mu+\nu+1}{2}\right) \Gamma\left(\frac{\mu-\nu+1}{2}\right), \\
\operatorname{Re}(\mu+\nu)>-1, \operatorname{Re} \mu<1 / 2
\end{gathered}
$$

with $\mu=\nu$, we see that the inner integral on the right hand side of (35) equals 0 . Hence,

$$
\begin{aligned}
\int_{\rightarrow 0}^{\rightarrow \infty} x^{\nu-1 / 2}\left[\frac{d^{2}}{d x^{2}}+\frac{1}{x^{2}}\left(\frac{1}{4}-\nu^{2}\right)\right]^{n} f(x) d x=0, & n=0,1, \ldots,-1 / 2<\operatorname{Re} \nu<0, \\
(37) & n=1,2, \ldots, 0 \leqslant \operatorname{Re} \nu<1 / 2 .
\end{aligned}
$$

(b) Suppose now that $f$ satisfies conditions (i)-(vi) of Theorem 1. Then $\left[d^{2} / d x^{2}+\right.$ $\left.\left(1 / x^{2}\right)\left((1 / 4)-\nu^{2}\right)\right]^{n} f(x), \quad n=0,1, \ldots$, belongs to $L_{2}\left(R_{+}\right)$.

(b-i) Let $-1 / 2<\operatorname{Re} \nu<0$ and $g_{n}(y)$ be the H-transforms $H_{\nu}, \quad-1 / 2<\operatorname{Re} \nu<$ 0 , of $\left[d^{2} / d x^{2}+\left(1 / x^{2}\right)\left((1 / 4)-\nu^{2}\right)\right]^{n} f(x), \quad n=0,1, \ldots$ Then

$$
g_{n}(y)=\int_{0}^{\infty} \sqrt{x y} \mathbf{H}_{\nu}(x y)\left[\frac{d^{2}}{d x^{2}}+\frac{1}{x^{2}}\left(\frac{1}{4}-\nu^{2}\right)\right]^{n} f(x) d x, \quad n=0,1,2, \ldots,
$$


where the integrals are understood in the $L_{2}\left(R_{+}\right)$norm. Put

$$
g_{n}^{N}(y)=\int_{1 / N}^{N} \sqrt{x y} \mathbf{H}_{\nu}(x y)\left[\frac{d^{2}}{d x^{2}}+\frac{1}{x^{2}}\left(\frac{1}{4}-\nu^{2}\right)\right]^{n} f(x) d x, \quad n=0,1,2 \ldots
$$

Then $g_{n}^{N}(y)$ tends to $g_{n}(y)$ in $L_{2}$ norm as $N \rightarrow \infty$. Let $n \geqslant 1$. Integrating (39) by parts twice, we obtain

$$
\begin{aligned}
& g_{n}^{N}(y)=\left.\left\{\sqrt{x y H_{\nu}(x y)} \frac{d}{d x}\left[\frac{d^{2}}{d x^{2}}+\frac{1}{x^{2}}\left(\frac{1}{4}-\nu^{2}\right)\right]^{n-1} f(x)\right\}\right|_{x=1 / N} ^{x=N} \\
& -\left.\left\{\frac{\partial}{\partial x}\left(\sqrt{x y} \mathbf{H}_{\nu}(x y)\right)\left[\frac{d^{2}}{d x^{2}}+\frac{1}{x^{2}}\left(\frac{1}{4}-\nu^{2}\right)\right]^{n-1} f(x)\right\}\right|_{x=1 / N} ^{x=N}
\end{aligned}
$$

(40)

$$
+\int_{1 / N}^{N}\left[\frac{\partial^{2}}{\partial x^{2}}+\frac{1}{x^{2}}\left(\frac{1}{4}-\nu^{2}\right)\right]\left(\sqrt{x y} \mathbf{H}_{\nu}(x y)\right)\left[\frac{d^{2}}{d x^{2}}+\frac{1}{x^{2}}\left(\frac{1}{4}-\nu^{2}\right)\right]^{n-1} f(x) d x .
$$

Using formulas [1]

(41)

$$
\frac{\partial}{\partial x}\left(\sqrt{x y} \mathbf{H}_{\nu}(x y)\right)=(1 / 2-\nu) \sqrt{\frac{y}{x}} \mathbf{H}_{\nu}(x y)+y \sqrt{x y} \mathbf{H}_{\nu-1}(x y)
$$

$\left[\frac{\partial^{2}}{\partial x^{2}}+\frac{1}{x^{2}}\left(\frac{1}{4}-\nu^{2}\right)\right]\left(\sqrt{x y} \mathbf{H}_{\nu}(x y)\right)=\frac{2^{1-\nu} y^{\nu+3 / 2}}{\sqrt{\pi} \Gamma(\nu+1 / 2)} x^{\nu-1 / 2}-y^{2} \sqrt{x y} \mathbf{H}_{\nu}(x y)$,

we have

(42)

$$
\begin{aligned}
g_{n}^{N}(y) & =\sqrt{N y} \mathbf{H}_{\nu}(N y) \frac{d}{d x}\left[\frac{d^{2}}{d x^{2}}+\frac{1}{x^{2}}\left(\frac{1}{4}-\nu^{2}\right)\right]^{n-1} f(N) \\
& -\sqrt{\frac{y}{N}} \mathbf{H}_{\nu}(y / N) \frac{d}{d x}\left[\frac{d^{2}}{d x^{2}}+\frac{1}{x^{2}}\left(\frac{1}{4}-\nu^{2}\right)\right]^{n-1} f(1 / N) \\
& +\left(\nu-\frac{1}{2}\right) \sqrt{\frac{y}{N}} \mathbf{H}_{\nu}(N y)\left[\frac{d^{2}}{d x^{2}}+\frac{1}{x^{2}}\left(\frac{1}{4}-\nu^{2}\right)\right]^{n-1} f(N) \\
& -y \sqrt{N y} \mathbf{H}_{\nu-1}(N y)\left[\frac{d^{2}}{d x^{2}}+\frac{1}{x^{2}}\left(\frac{1}{4}-\nu^{2}\right)\right]^{n-1} f(N) \\
& +\left(\frac{1}{2}-\nu\right) \sqrt{N y} \mathbf{H}_{\nu}(y / N)\left[\frac{d^{2}}{d x^{2}}+\frac{1}{x^{2}}\left(\frac{1}{4}-\nu^{2}\right)\right]^{n-1} f(1 / N)
\end{aligned}
$$




$$
\begin{aligned}
& +y \sqrt{\frac{y}{N}} \mathbf{H}_{\nu-1}(y / N)\left[\frac{d^{2}}{d x^{2}}+\frac{1}{x^{2}}\left(\frac{1}{4}-\nu^{2}\right)\right]^{n-1} f(1 / N) \\
& -y^{2} \int_{1 / N}^{N} \sqrt{x y} \mathbf{H}_{\nu}(x y)\left[\frac{d^{2}}{d x^{2}}+\frac{1}{x^{2}}\left(\frac{1}{4}-\nu^{2}\right)\right]^{n-1} f(x) d x \\
& +\frac{2^{1-\nu} y^{\nu+3 / 2}}{\sqrt{\pi} \Gamma(\nu+1 / 2)} \int_{1 / N}^{N} x^{\nu-1 / 2}\left[\frac{d^{2}}{d x^{2}}+\frac{1}{x^{2}}\left(\frac{1}{4}-\nu^{2}\right)\right]^{n-1} f(x) d x .
\end{aligned}
$$

Here $P(d / d x) f(N)$ means $\left.P(d / d x) f(x)\right|_{x=N}$. As $N$ tends to infinity, integral (49) vanishes because of property (vi). Applying the asymptotic formula for the Struve function $[\mathbf{1}]$

$$
\mathbf{H}_{\nu}(y)= \begin{cases}O\left(y^{-1 / 2}\right), & y \rightarrow \infty, \quad \mathcal{R} e \nu<1 / 2 \\ O\left(y^{\mathcal{R} e^{2}+1}\right), & y \rightarrow 0, \quad \forall \nu\end{cases}
$$

we obtain that $\sqrt{N y} \mathbf{H}_{\nu}(N y),|\mathcal{R} e \nu|<1 / 2$, is uniformly bounded. The function $(d / d x)\left[d^{2} / d x^{2}+\left(1 / x^{2}\right)\left((1 / 4)-\nu^{2}\right)\right]^{n-1} f(N)$ tends to 0 as $N$ approaches infinity (property (v)), therefore, the expression on the right hand side of (42) tends to 0 as $N$ approaches infinity. From (iv) we see that $(d / d x)\left[d^{2} / d x^{2}+\left(1 / x^{2}\right)\left((1 / 4)-\nu^{2}\right)\right]^{n-1}$ $f(1 / N)$ has order $O(N)$, whereas function $\sqrt{y / N} \mathbf{H}_{\nu}(y / N)$ has order $O\left(N^{-3 / 2-\nu}\right)$. Hence, expression (43) approaches 0 as $N$ tends to infinity. Function $\sqrt{y / N} \mathbf{H}_{\nu}(N y)$ has order $O\left(N^{-1}\right)$, whereas the expression $\left[d^{2} / d x^{2}+\left(1 / x^{2}\right)\left((1 / 4)-\nu^{2}\right)\right]^{n-1} f(N)$ is $o(1)$ (property (iii)), therefore, expression (44) is $o$ (1). The function $y \sqrt{N y} \mathbf{H}_{\nu-1}(N y)$ is $O(1)$, hence, property (iii) shows that (45) is $o(1)$. Since $\sqrt{N y} \mathbf{H}_{\nu}(y / N)$ has the order $O\left(N^{-1 / 2-\nu}\right)$, and $\left[d^{2} / d x^{2}+\left(1 / x^{2}\right)\left((1 / 4)-\nu^{2}\right)\right]^{n-1} f(1 / N)$ is $o(1)$ (property (iii)), expression (46) is also $o(1)$. The function $y \sqrt{y / N} \mathbf{H}_{\nu-1}(y / N)$ has the order $O\left(N^{-1 / 2-\nu}\right)$, hence, property (iii) shows that (47) is $o(1)$.

Therefore, the right hand side of (42), as well as all functions (43) - (49), except (48), vanish as $N$ tends to infinity, whereas expression (48) converges to $-y^{2} g_{n-1}(y)$. Consequently, $g_{n}(y)=-y^{2} g_{n-1}(y)$, and therefore, $g_{n}(y)=\left(-y^{2}\right)^{n} g_{0}(y), \quad n=$ $0,1, \ldots$ Thus $g(y)=g_{0}(y)$ such that $y^{2 n} g(y) \in L_{2}\left(R_{+}\right), \quad n=0,1, \ldots$, is the $\mathbf{H}$ transform $\mathbf{H}_{\nu}$ of the function $f(x)$. But the $\mathbf{H}$-transform $\mathbf{H}_{\nu}$ is the inverse of the Y-transform $Y_{\nu}$ if $-1 / 2<\mathcal{R} e \nu<0$, so we obtain that and $f$ is the $Y$-transform $Y_{\nu},-1 / 2<\mathcal{R} e \nu<0$, of a function $g$ such that $y^{n} g(y) \in L_{2}\left(R_{+}\right), \quad n=0,1, \ldots$.

(b-ii) Let now $0<\operatorname{Re} \nu<1 / 2$. The inverse of the $\mathrm{Y}$-transform $Y_{\nu}$ in the range $0<\operatorname{Re} \nu<1$ has the form [3]

$$
g(y)=y^{-\nu-1 / 2} \frac{d}{d y} y^{\nu+1 / 2} \int_{0}^{\infty} \sqrt{x y}\left[\mathbf{H}_{\nu+1}(x y)-\frac{(x y)^{\nu}}{2^{\nu} \sqrt{\pi} \Gamma(\nu+3 / 2)}\right] f(x) d x, \quad y \in R_{+},
$$


that can be expressed in an equivalent form

$$
g(y)=\lim _{N \rightarrow \infty} \int_{1 / N}^{N}\left[\sqrt{x y} \mathbf{H}_{\nu}(x y)-\frac{(x y)^{\nu-1 / 2}}{2^{\nu-1} \sqrt{\pi} \Gamma(\nu+1 / 2)}\right] f(x) d x, \quad y \in R_{+},
$$

where the limit is understood in the $L_{2}\left(R_{+}\right)$norm. Putting

$$
\begin{array}{r}
g_{n}^{N}(y)=\int_{1 / N}^{N}\left[\sqrt{x y} \mathbf{H}_{\nu}(x y)-\frac{(x y)^{\nu-1 / 2}}{2^{\nu-1} \sqrt{\pi} \Gamma(\nu+1 / 2)}\right]\left[\frac{d^{2}}{d x^{2}}+\frac{1}{x^{2}}\left(\frac{1}{4}-\nu^{2}\right)\right]^{n} f(x) d x \\
n=0,1,2 \ldots,
\end{array}
$$

we see that $g_{n}^{N}(y)$ tends to some functions $g_{n}(y)$ in the $L_{2}$ norm as $N \rightarrow \infty$. Let $n \geqslant 1$. Integrating (53) by parts twice and using formulae (40), (41) we obtain

$$
\begin{aligned}
g_{n}^{N}(y) & =\sqrt{N y} \mathbf{H}_{\nu}(N y) \frac{d}{d x}\left[\frac{d^{2}}{d x^{2}}+\frac{1}{x^{2}}\left(\frac{1}{4}-\nu^{2}\right)\right]^{n-1} f(N) \\
& -\sqrt{\frac{y}{N}} \mathbf{H}_{\nu}(y / N) \frac{d}{d x}\left[\frac{d^{2}}{d x^{2}}+\frac{1}{x^{2}}\left(\frac{1}{4}-\nu^{2}\right)\right]^{n-1} f(1 / N) \\
& +\left(\nu-\frac{1}{2}\right) \sqrt{\frac{y}{N}} \mathbf{H}_{\nu}(N y)\left[\frac{d^{2}}{d x^{2}}+\frac{1}{x^{2}}\left(\frac{1}{4}-\nu^{2}\right)\right]^{n-1} f(N) \\
& -y \sqrt{N y} \mathbf{H}_{\nu-1}(N y)\left[\frac{d^{2}}{d x^{2}}+\frac{1}{x^{2}}\left(\frac{1}{4}-\nu^{2}\right)\right]^{n-1} f(N) \\
& +\left(\frac{1}{2}-\nu\right) \sqrt{N y} \mathbf{H}_{\nu}(y / N)\left[\frac{d^{2}}{d x^{2}}+\frac{1}{x^{2}}\left(\frac{1}{4}-\nu^{2}\right)\right]^{n-1} f(1 / N) \\
& +y \sqrt{\frac{y}{N}} \mathbf{H}_{\nu-1}(y / N)\left[\frac{d^{2}}{d x^{2}}+\frac{1}{x^{2}}\left(\frac{1}{4}-\nu^{2}\right)\right]^{n-1} f(1 / N) \\
& -y^{2} \int_{1 / N}^{N} \sqrt{x y}\left[\mathbf{H}_{\nu}(x y)-\frac{2^{1-\nu}(x y)^{\nu-1}}{\sqrt{\pi} \Gamma(\nu+1 / 2)}\right] \\
& {\left[\frac{d^{2}}{d x^{2}}+\frac{1}{x^{2}}\left(\frac{1}{4}-\nu^{2}\right)\right]^{n-1} f(x) d x } \\
& -\frac{2^{1-\nu} y^{\nu-1 / 2}}{\sqrt{\pi} \Gamma(\nu+1 / 2)} \int_{1 / N}^{N} x^{\nu-1 / 2}\left[\frac{d^{2}}{d x^{2}}+\frac{1}{x^{2}}\left(\frac{1}{4}-\nu^{2}\right)\right]^{n} f(x) d x
\end{aligned}
$$

When $N$ tends to infinity integral (61) vanishes because of property (vi) and $n \geqslant 1$. Reasoning the same as before, we can conclude that the right hand side of (54), as well as all functions (55)-(59), vanish as $N$ tends to infinity, whereas the expression (60) 
converges to $-y^{2} g_{n-1}(y)$. Consequently, $g_{n}(y)=-y^{2} g_{n-1}(y)$, and therefore, $g_{n}(y)=$ $\left(-y^{2}\right)^{n} g_{0}(y), \quad n=0,1, \ldots$. Thus $g(y)=g_{0}(y)$ such that $y^{2 n} g(y) \in L_{2}\left(R_{+}\right), \quad n=$ $0,1, \ldots$, is the transform (52) of function $f(x)$. But transform (52) is the inverse of the Y-transform $Y_{\nu}$ if $0<\mathcal{R} e \nu<1 / 2$, so we obtain that and $f$ is the Y-transform $Y_{\nu}, \quad 0<\mathcal{R} e \nu<1 / 2$, of a function $g$ such that $y^{n} g(y) \in L_{2}\left(R_{+}\right), \quad n=0,1, \ldots$ Theorem 1 is thus proved.

REMARK. The case $\operatorname{Re} \nu=0$ has been excluded from Theorem 1. It was proved in [3] that in this case the range of the Y-transform in $L_{2}\left(R_{+}\right)$is a proper subspace of $L_{2}\left(R_{+}\right)$.

\section{Y-TRANSFORM OF SQUARE INTEGRABLE FUNCTIONS WITH COMPACT SUPPORTS}

Now we describe the Y-transform of square integrable functions with compact supports (the Paley-Wiener theorem for the Y-transform).

THEOREM 2. A function $f$ is the $Y$-transform $Y_{\nu}, 0<|\mathcal{R e} \nu|<1 / 2$, of a square integrable function $g$ with compact support on $[0, \infty)$ if and only if $f$ satisfies conditions (i)-(vi) of Theorem 1 and moreover,

$$
\lim _{n \rightarrow \infty}\left\|\left[\frac{d^{2}}{d x^{2}}+\frac{1}{x^{2}}\left(\frac{1}{4}-\nu^{2}\right)\right]^{n} f(x)\right\|_{2}^{1 /(2 n)}=\sigma_{g}<\infty,
$$

where

$$
\sigma_{g}=\sup \{y: y \in \operatorname{supp} g\},
$$

and the support of a function is the smallest closed set outside which the function vanishes almost everywhere [12].

Proof: (a) Let $f(x)$ be the Y-transform of $g(y) \in L_{2}\left(R_{+}\right)$and $\sigma_{g}<\infty$ :

$$
f(x)=\int_{0}^{\sigma_{g}} \sqrt{x y} Y_{\nu}(x y) g(y) d y, \quad 0<|\mathcal{R} e \nu|<1 / 2 .
$$

One can assume that $\sigma_{g}>0$, otherwise it is trivial. Since $\sigma_{g}<\infty$ we have $y^{n} g(y) \in$ $L_{2}\left(R_{+}\right)$for all $n=0,1,2, \ldots$ Therefore, $f$ satisfies conditions (i)-(vi) of Theorem 1. Furthermore,

$$
\left[\frac{d^{2}}{d x^{2}}+\frac{1}{x^{2}}\left(\frac{1}{4}-\nu^{2}\right)\right]^{n} f(x)=\int_{0}^{\sigma_{g}} \sqrt{x y} Y_{\nu}(x y)\left(-y^{2}\right)^{n} g(y) d y .
$$

Consequently, applying the inequality (2) for the Y-transform (65), we obtain

$$
\left\|\left[\frac{d^{2}}{d x^{2}}+\frac{1}{x^{2}}\left(\frac{1}{4}-\nu^{2}\right)\right]^{n} f(x)\right\|_{2}^{2} \leqslant C \int_{0}^{\sigma_{g}} y^{4 n}|g(y)|^{2} d y \leqslant C \sigma_{g}^{4 n} \int_{0}^{\sigma_{g}}|g(y)|^{2} d y .
$$




\section{Hence,}

(67)

$\varlimsup_{n \rightarrow \infty}\left\|\left[\frac{d^{2}}{d x^{2}}+\frac{1}{x^{2}}\left(\frac{1}{4}-\nu^{2}\right)\right]^{n} f(x)\right\|_{2}^{1 /(2 n)} \leqslant \varlimsup_{n \rightarrow \infty} C^{1 /(4 n)} \sigma_{g}\left\{\int_{0}^{\sigma_{g}}|g(y)|^{2} d y\right\}^{1 /(4 n)}=\sigma_{g}$.

On the other hand, since $\sigma_{g}$ is the least upper bound of the support of $g$, for every $\varepsilon, 0<\varepsilon<\sigma_{g}$, we have

$$
\int_{\sigma_{g}-\varepsilon}^{\sigma_{g}}|g(y)|^{2} d y>0
$$

Consequently, using now inequality (3) for the Y-transform (65), we get

$\lim _{n \rightarrow \infty}\left\|\left[\frac{d^{2}}{d x^{2}}+\frac{1}{x^{2}}\left(\frac{1}{4}-\nu^{2}\right)\right]^{n} f(x)\right\|_{2}^{1 /(2 n)} \geqslant \varliminf_{n \rightarrow \infty} C^{-1 /(4 n)}\left\{\int_{\sigma_{g}-\varepsilon}^{\sigma_{g}} y^{4 n}|g(y)|^{2} d y\right\}^{1 /(4 n)}$

$$
\geqslant\left(\sigma_{g}-\varepsilon\right) \varliminf_{n \rightarrow \infty} C^{-1 /(4 n)}\left\{\int_{\sigma_{g}-\varepsilon}^{\sigma_{g}}|g(y)|^{2} d y\right\}^{1 /(4 n)}=\sigma_{g}-\varepsilon .
$$

Because $\varepsilon$ can be chosen arbitrarily small, from (69) and (67) we obtain (62).

(b) Suppose now that $f$ satisfies the conditions of Theorem 1, and the limit in (62) exists and equals $\sigma<\infty$. Applying Theorem 1 we see that $f$ is the Y-transform $Y_{\nu}$ of a function $g$ with $\sigma_{g}$ defined by (63) such that $y^{n} g(y) \in L_{2}\left(R_{+}\right), \quad n=0,1, \ldots$ We prove that $\sigma_{g}<\infty$ and moreover, $\sigma=\sigma_{g}$. Theorem 1 implies that (11) holds. Therefore, using inequalities (2) and (3) we obtain

$$
C^{-1}\left\|y^{2 n} g(y)\right\|_{2} \leqslant\left\|\left[\frac{d^{2}}{d x^{2}}+\frac{1}{x^{2}}\left(\frac{1}{4}-\nu^{2}\right)\right]^{n} f(x)\right\|_{2} \leqslant C\left\|y^{2 n} g(y)\right\|_{2} .
$$

Hence,

$$
\begin{aligned}
\lim _{n \rightarrow \infty} C^{-1 /(2 n)}\left\|y^{2 n} g(y)\right\|_{2}^{1 /(2 n)} & \leqslant \lim _{n \rightarrow \infty}\left\|\left[\frac{d^{2}}{d x^{2}}+\frac{1}{x^{2}}\left(\frac{1}{4}-\nu^{2}\right)\right]^{n} f(x)\right\|_{2}^{1 /(2 n)}=\sigma \\
& \leqslant \lim _{n \rightarrow \infty} C^{1 /(2 n)}\left\|y^{2 n} g(y)\right\|_{2}^{1 /(2 n)} .
\end{aligned}
$$

Consequently,

$$
\lim _{n \rightarrow \infty}\left\|y^{2 n} g(y)\right\|_{2}^{1 /(2 n)}=\sigma .
$$

Suppose that $\sigma_{g}>\sigma$. Then there exists a positive $\varepsilon$ such that

$$
\int_{\sigma+\varepsilon}^{\infty}|g(y)|^{2} d y>0
$$


We have

$$
\begin{aligned}
\sigma=\lim _{n \rightarrow \infty}\left\|y^{2 n} g(y)\right\|_{2}^{1 /(2 n)} & \geqslant \lim _{n \rightarrow \infty}\left\{\int_{\sigma+\varepsilon}^{\infty} y^{4 n}|g(y)|^{2} d y\right\}^{1 /(4 n)} \\
& \geqslant(\sigma+\varepsilon) \lim _{n \rightarrow \infty}\left\{\int_{\sigma+e}^{\infty}|g(y)|^{2} d y\right\}^{1 /(4 n)}=\sigma+\varepsilon .
\end{aligned}
$$

This is impossible. Hence, $\sigma_{g} \leqslant \sigma$ and therefore, the function $g$ has a compact support. Suppose now that $\sigma_{g}<\sigma$. Then there exists a positive $\varepsilon$ such that

$$
\int_{\sigma-\varepsilon}^{\infty}|g(y)|^{2} d y=0
$$

We have

$$
\begin{aligned}
\sigma=\lim _{n \rightarrow \infty}\left\|y^{2 n} g(y)\right\|_{2}^{1 /(2 n)} & \leqslant \overline{\lim }_{n \rightarrow \infty}\left\{\int_{0}^{\sigma-\varepsilon} y^{4 n}|g(y)|^{2} d y\right\}^{1 /(4 n)} \\
& \leqslant(\sigma-\varepsilon) \varlimsup_{n \rightarrow \infty}\left\{\int_{0}^{\sigma-\varepsilon}|g(y)|^{2} d y\right\}^{1 /(4 n)}=\sigma-\varepsilon
\end{aligned}
$$

This is also impossible. Hence, $\sigma_{g} \geqslant \sigma$, and consequently, $\sigma_{g}=\sigma<\infty$. Theorem 2 is thus proved.

REMARK. If a function $f$ satisfies conditions of Theorem 1 , then the limit (62) always exists. It equals to infinity if $f$ is the Y-transform $Y_{\nu}$ of a function $g$ with unbounded support.

\section{Y-TRANSFORM OF ANALYTIC FUNCTIONS}

We consider now the Y-transform $Y_{\nu}$ of functions analytic in some angle.

Theorem 3. The $Y$-transform $Y_{\nu},-1<\mathcal{R} e \nu<1$, maps the space of all functions $g(z)$, regular in the angle $-\alpha<\arg z<\beta$, where $0<\alpha, \beta \leqslant \pi$; of the order $O\left(|z|^{-a-\varepsilon}\right)$ for small $z$, and $O\left(|z|^{-b+e}\right)$ for large $z$, where $a<1 / 2<b$, uniformly for any small positive $\varepsilon$ in any angle interior to the above; and satisfying conditions

$$
\int_{0}^{\infty} y^{\nu+2 n+1 / 2} g(y) d y=0, \quad n \in(-b / 2-\mathcal{R} e \nu / 2-1 / 4,-a / 2-\mathcal{R} e \nu / 2-1 / 4),
$$

$$
\int_{0}^{\infty} y^{-\nu+2 n+1 / 2} g(y) d y=0, \quad n \in(-b / 2+\operatorname{Re} \nu / 2-1 / 4,-a / 2+\operatorname{Re} \nu / 2-1 / 4),
$$

for all nonnegative integers $n$, if there exists such $n$, one-to-one onto the space of all functions $f(z)$, regular in the angle $-\beta<\arg z<\alpha$, of the order $O\left(|z|^{1-b-\varepsilon}\right)$ for 
small $z$, and $O\left(|z|^{1-a+e}\right)$ for large $z$, uniformly for any small positive $\varepsilon$ in any angle interior to the above; and satisfying conditions

$$
\int_{0}^{\infty} x^{\nu-2 n-1 / 2} f(x) d x=0, \quad n \in(a / 2+\mathcal{R} e \nu / 2-1 / 4, b / 2+\mathcal{R} e \nu / 2-1 / 4)
$$

(78)

$$
\int_{0}^{\infty} x^{\nu+2 n+3 / 2} f(x) d x=0, \quad n \in(-b / 2-\mathcal{R} e \nu / 2-3 / 4,-a / 2-\mathcal{R} e \nu / 2-3 / 4)
$$

for all nonnegative integers $n$, if there exists such $n$. (For example, if $\operatorname{Re} \nu=0$, then $n=0$ always belongs to the interval $(a / 2-1 / 4, b / 2-1 / 4)$.)

ProOF: Let $g(z)$ satisfy the conditions of Theorem 3. Then the function $g(z)$ on $R_{+}$belongs to $L_{2}\left(R_{+}\right)$and its Mellin transform $g^{*}(s)$

$$
g^{*}(s)=\int_{0}^{\infty} x^{s-1} g(x) d x
$$

is an analytic function of $s$, regular for $a<\mathcal{R} e s<b$; and

$$
g^{*}(s)= \begin{cases}O\left(e^{-(\beta-\varepsilon) \mathcal{I} m s}\right), & \mathcal{I} m s \rightarrow \infty \\ O\left(e^{(\alpha-\varepsilon) \mathcal{I} m s}\right), & \mathcal{I} m s \rightarrow-\infty\end{cases}
$$

for every positive $\varepsilon$, uniformly in any strip interior to $a<\mathcal{R e} s<b$ (see [8]). Let $f(x)$ be the Y-transform $Y_{\nu},-1<\mathcal{R} e \nu<1$, of $g(y)$. Since $g(y)$ belongs to $L_{2}\left(R_{+}\right)$, the Parseval identity for the $\mathrm{Y}$-transform $Y_{\nu}$ holds on the line $\operatorname{Re} s=1 / 2$ [6]:

$$
f^{*}(s)=2^{s-1} \frac{\Gamma\left(\frac{1}{4}+\frac{\nu}{2}+\frac{s}{2}\right) \Gamma\left(\frac{1}{4}-\frac{\nu}{2}+\frac{s}{2}\right)}{\Gamma\left(-\frac{1}{4}-\frac{\nu}{2}+\frac{s}{2}\right) \Gamma\left(\frac{5}{4}+\frac{\nu}{2}-\frac{s}{2}\right)} g^{*}(1-s) .
$$

Because of $(77)$ the function $g^{*}(1-s)$ equals 0 at the poles of function $\Gamma(1 / 4+\nu / 2+s / 2)$ $\Gamma(1 / 4-\nu / 2+s / 2)$ in the strip $1-b<\mathcal{R} e s<1-a$, if there exists one. Hence, from (81) one can see that $f^{*}(s)$ is analytic in the strip $1-b<\mathcal{R} e s<1-a$. Furthermore, since the function $2^{s-1 / 2}(\Gamma(1 / 4+\nu / 2+s / 2) \Gamma(1 / 4-\nu / 2+s / 2)) /(\Gamma(-1 / 4-\nu / 2+s / 2)$ $\Gamma(5 / 4+\nu / 2-s / 2))$ is uniformly bounded in any compact domain in the strip $1-b<$ $\mathcal{R} e s<1-a$, not containing the poles of function $\Gamma(1 / 4+\nu / 2+s / 2) \Gamma(1 / 4-\nu / 2+s / 2)$, and has at most only polynomial growth as $\mathcal{I} m s \rightarrow \pm \infty$, from (80) we see that function $f^{*}(s)$ decays exponentially

$$
f^{*}(s)= \begin{cases}O\left(e^{(\beta-\varepsilon) \mathcal{I} m s}\right), & \mathcal{I} m s \rightarrow-\infty \\ O\left(e^{-(\alpha-\varepsilon) \mathcal{I} m}\right), & \operatorname{Im} s \rightarrow \infty\end{cases}
$$


for every positive $\varepsilon$, uniformly in any strip interior to $1-b<\mathcal{R e} s<1-a$. Hence, its inverse Mellin transform $f(z)$ is regular for $-\beta<\arg z<\alpha$, and of the order $O\left(|z|^{b-1-\varepsilon}\right)$ for small $z$, and $O\left(|z|^{a-1+\varepsilon}\right)$ for large $z$, uniformly in any angle interior to the above angle for any small positive $\varepsilon[8]$. Moreover, $f^{*}(s)$ has zeros at the poles of the function $\Gamma(-1 / 4-\nu / 2+s / 2) \Gamma(5 / 4+\nu / 2-s / 2)$ in the strip $1-b<\operatorname{Re} s<1-a$, if there exists one. Hence (78) holds.

Conversely, let $f(z)$ satisfy the conditions of Theorem 3 . Then $f(z)$ on $R_{+}$belongs to $L_{2}\left(R_{+}\right)$and its Mellin transform (79) $f^{*}(s)$ is analytic in the strip $1-b<\mathcal{R} e s<$ $1-a$ and satisfies (82). Furthermore, because of (78) the function $f^{*}(s)$ vanishes at the poles of the function $\Gamma(-1 / 4-\nu / 2+s / 2) \Gamma(5 / 4+\nu / 2-s / 2)$ in the strip $1-b<$ $\mathcal{R} e s<1-a$, if there exists one. Therefore, if we express $f^{*}(s)$ in the form (81), function $g^{*}(s)$ is analytic in the strip $a<\mathcal{R} e s<b$; and has the asymptotics (80) for every positive $\varepsilon$, uniformly in any strip interior to $a<\mathcal{R} e s<b$. Furthermore, $g^{*}(1-s)$ has zeros at the poles of the function $\Gamma(1 / 4+\nu / 2+s / 2) \Gamma(1 / 4-\nu / 2+s / 2)$ in the strip $1-b<\operatorname{Re} s<1-a$. Consequently, the inverse Mellin transform $g(z)$ of $g^{*}(s)$ satisfies the conditions of Theorem 3 and $f$ is the Y-transform of $g$.

If in Theorem 3 we take $\alpha=\beta$ and $0<a<\min \{|\nu|,|\nu+1|,|\nu-1|\}$, then in the strip $1 / 2-a<\operatorname{Re} s<1 / 2+a$ there are no poles or zeros of the function $2^{s-1 / 2}(\Gamma(1 / 4+\nu / 2+s / 2) \Gamma(1 / 4-\nu / 2+s / 2)) /(\Gamma(-1 / 4-\nu / 2+s / 2) \Gamma(5 / 4+\nu / 2-$ $s / 2)$ ), hence, we have

Corollary 1. The Y-transform $Y_{\nu}, 0<|\mathcal{R e} \nu|<1$, is a bijection in the space of all functions, regular in the angle $|\arg z|<\alpha$, where $0<\alpha \leqslant \pi$; of order $O\left(|z|^{a-1 / 2-\varepsilon}\right)$ for small $z$, and $O\left(|z|^{-a-1 / 2+\varepsilon}\right)$ for large $z$, uniformly for any small positive $\varepsilon, 0<\varepsilon<a$, in any angle interior to the above, where $0<a<$ $\min \{|\nu|,|\nu+1|,|\nu-1|\}$.

\section{Y-TRANSFORM IN SOME OTHER SPACES OF FUNCTIONS}

In $[9,10]$ the Y-transform is proved to be a bijection in some spaces of functions $\mathcal{M}_{c, \gamma}^{-1}(L)$ introduced there. In this section the $\mathrm{Y}$-transform in a space of functions including the spaces $\mathcal{M}_{c, \gamma}^{-1}(L)$ as special cases is considered.

Let $\Phi$ be any linear subspace of either $L_{1}(R)$ or $L_{2}(R)$ having properties:

(i) if $\phi(t) \in \Phi$ then $\phi(-t) \in \Phi$;

(ii) functions $\varphi(t)=2^{i t} \Gamma(1 / 2+\nu / 2+i t / 2) \Gamma(1 / 2-\nu / 2+i t / 2) \sin (\pi / 2)$ (it $-\nu), 0<|\operatorname{Re} \nu|<1$, and $\varphi^{-1}(t)$ are multipliers of $\Phi$.

It is easy to see that $\varphi^{-1}(-t)$ is also a multiplier of $\Phi$. The multipliers $\varphi(t)$ and $\varphi^{-1}(t)$ are infinitely differentiable and uniformly bounded on $R$, and their derivatives 
grow logarithmically. Therefore, many classical spaces on $R$ are special cases of $\Phi$ (for example, any $L_{1}$ or $L_{2}$ space with $L_{\infty}$-weights, the Schwartz space $\mathcal{S}(R)$, and the space of infinitely differentiable functions with compact support [12]). On $R_{+}$we define $\mathcal{M}^{-1}(\Phi)$ to be the space of all functions $g$ that can be represented in the form

$$
g(x)=\int_{-\infty}^{\infty} \phi(t) x^{i t-1 / 2} d t
$$

almost everywhere, where $\phi \in \Phi$ (if $\phi \notin L_{1}(R)$ the integral should be understood as the inverse Mellin transform in $\left.L_{2}[\mathbf{8}]\right)$. The spaces $\mathcal{M}_{c, \gamma}^{-1}(L)[\mathbf{1 0}]$ as well as the space of functions considered in Corollary 1 are special cases of $\mathcal{M}^{-1}(\Phi)$.

Theorem 4. The $Y$-transform $Y_{\nu}, 0<|\mathcal{R} e \nu|<1$, is a bijection in $\mathcal{M}^{-1}(\Phi)$.

Proof: From (83) we see that if $g \in \mathcal{M}^{-1}(\Phi)$ then $g$ can be expressed in the form of the inverse Mellin transform

$$
g(x)=\frac{1}{2 \pi i} \int_{1 / 2-i \infty}^{1 / 2+i \infty} g^{*}(s) x^{-s} d s,
$$

where $g^{*}(1 / 2+i t) \in \Phi$. Using formula (36) we obtain that the Mellin transform (79) of the function $k(x)=\sqrt{x} Y_{\nu}(x)$ is $k^{*}(s)=\varphi(i / 2-i s)$. Applying the Parseval equation for the Mellin transform

$$
\int_{0}^{\infty} k(x y) g(y) d y=\frac{1}{2 \pi i} \int_{1 / 2-i \infty}^{1 / 2+i \infty} k^{*}(s) g^{*}(1-s) x^{-s} d s, \quad 0<|\operatorname{Re} \nu|<1,
$$

that has been proved for $g^{*}(1 / 2+i t) \in L_{2}(R)$ in [8] and $g^{*}(1 / 2+i t) \in L_{1}(R)$ in [9], we obtain

$$
\left(Y_{\nu} g\right)(x)=\int_{0}^{\infty} \sqrt{x y} Y_{\nu}(x y) g(y) d y=\frac{1}{2 \pi} \int_{-\infty}^{\infty} \varphi(t) g^{*}(1 / 2-i t) x^{-i t-1 / 2} d t
$$

Since $\varphi(t)$ and $\varphi^{-1}(-t)$ are multipliers of $\Phi$, then $\varphi(t) g^{*}(1 / 2-i t)$ belongs to $\Phi$ if and only if $g^{*}(1 / 2+i t)$ belongs to $\Phi$. Therefore, $\left(Y_{\nu} g\right)(x) \in \mathcal{M}^{-1}(\Phi)$ if and only if $g \in \mathcal{M}^{-1}(\Phi)$. Theorem 4 is thus proved.

\section{REFERENCES}

[1] M. Abramovitz and I.A. Stegun, Handbook of nathematical functions, with formulas, graphs, and mathematical tables (Dover Publication, New York, 1972).

[2] H.H. Bang, 'A property of infinitely differentiable functions', Proc. Amer. Math. Soc. 108 (1990), 73-76. 
[3] P. Heywood and P.G. Rooney, 'On the Hankel and some related transformations', Canad. J. Math. 40 (1988), 989-1009.

[4] T. Koornwinder, 'A new proof of a Paley-Wiener type theorem for the Jacobi transform', Ark. Mat. 13 (1975), 145-159.

[5] R.E.A.C. Paley and N. Wiener, Fourier transforms in the complex domain, Colloq. Publ. (American Mathematical Society, Providence, RI, 1934).

[6] P.G. Rooney, 'On the $\mathcal{Y}_{\nu}$ and $\mathcal{H}_{\nu}$ transformations', Canad. J. Math. 32 (1980), 1021-1044.

[7] P.G. Rooney, 'On the range of an integral transformation', Canad. Math. Bull. 37 (1994), 545-548.

[8] E.C. Titchmarsh, Introduction to the theory of Fourier integrals (Chelsea Publishing Company, New York, 1986).

[9] V.K. Tuan, 'On the theory of generalized integral transforms in a certain function space', (translated from Russian), Soviet Math. Dokl. 33 (1986), 103-106.

[10] V.K. Tuan, O.I. Marichev and S.B. Yakubovich, 'Composition structure of integral transformations', (translated from Russian), Soviet Math. Dokl. 33 (1986), 166-170.

[11] V.K. Tuan, 'A real-variable approach to the Paley-Wiener theorem', (submitted).

[12] K. Yosida, Functional analysis, (Sixth Edition) (Springer-Verlag, Berlin, Heidelberg, New York, 1980).

Department of Mathematics and Computer Science

Kuwait University

P.O. Box 5969

Safat 13060

Kuwait 